far more rapidly with a change of composition than do the other properties, diffusivity, tensile strength, and Young's modulus, which make up the thermal endurance of the glass. The coefficient of expansion of German laboratory glassware is something more than Io per cent. lower than that of English, and this fact alone would account for the breakage of the English glass with drastic heat treatment.

There is no particular difficulty attached to the manufacture of glass having a low coefficient of expansion and high thermal endurance, and we may take it that the English manufacturers have decided that the maximum resistance to attack by reagents is to be desired, and have, accordingly, sacrificed thermal endurance to a smail extent in order to obtain the encouraging results outlined in Mr. Jenkinson's letter in NATURE of October 28.

The whole question is largely one of general policy. If the chemists of this country prefer to use a glass of higher thermal endurance but with less resistance to reagents, then I have little doubt that the British makers would supply it. $\quad$ E. A. COAD PrYor.

Milford, Park Road, Teddington, November 8.

\section{The Separation of the Element Chlorine into Normal Chlorine and Meta-Chlorine, and the Positive Electron.}

IN commenting on a letter under the above heading from Prof. Harkins in Nature of April 22 last, I remarked that his assumption that "the hydrogen nucleus or the positive electron has, according to these papers, a weight, and presumably a mass, of $\mathrm{I} \cdot 000$, on the basis of oxygen as 16.000," was contradicted by experiment.

Prof. Harkins has pointed out to me that this assumption was made with the reservation "whenever the positive electron is combined in a complex atom," which I was careless enough to overlook. I wish, therefore, to apologise for my remark and withdraw it unreservedly.

I very much regret that this apology comes so late, but the delay is due to the fact that Prof. Harkins led me to understand that he was himself publishing a statement on the matter in NATURE, to which I could reply. He now tells me he has decided not to do so.

Trinity College, Cambridge, November 9.

\section{The Stereoscopic Appearance of Gertain Pictures.}

IN going round a picture gallery it will be noticed that in certain pictures the objects delineated appear to stand out in a similar manner to those seen with a stereoscope. A picture of this kind has the effect of making those surrounding it appear very flat by comparison. This appearance is not the characteristic of a particular artist, because in an exhibition, all the paintings being by one man, only one or two may be found which have this stereoscopic appearance.

The majority of the pictures will be correct in drawing, perspective, light and shade, but it will be noticed that this will be correct only for one eyethat is to say, the picture is quite correct for either eye when the other is closed. Those pictures, however, which have a stereoscopic appearance are painted so that the representation is as nearly as possible a delineation as seen by the combined retinæ, any disturbing element which would do away with this illusion being eliminated. For instance, a revolver pointed straight at a person so that the centre of the barrel is pointing directly between the two eyes will be seen quite differently with the two eyes. When the right eye is closed the left-hand NO. 2664 , VOL. IO6] side of the barrel will be seen, the right-hand side being invisible. When the left eye is closed the righthand side is seen, and the left-hand side of the barrel is invisible. When, therefore, the barrel of the revolver is foreshortened and both sides are visible, the muzzle being pointed directly at the observer, the appearance is such as could be seen only with both eyes. The stereoscopic appearance is then very striking, and the revolver appears to follow the observer and to be pointed directly at him, no matter what position he takes up with regard to the picture. In all the pictures or portions of them which present this stereoscopic appearance it will be noticed that the appearance as seen by both eyes is represented, the left-hand side of the picture being represented as seen by the left eye, the right-hand side as seen by the right eye.

In a demonstration which $\mathrm{I}$ gave before the Physiological Society (Journal of Physiology, vol. xlviii., I914) I showed that the perception of binocular relief is independent of double images and the stimulation of disparate points, provided that the object presents images to the two retinæ similar to those which are presented by an object in the field of vision. This can be shown by taking a pair of stereoscopic photographs in which the point of sight is at the centre of each and cutting them vertically in two, and then, having pasted the left half of the left photograph on the left side and the right half of the right photograph on the right side on white or black cardboard at an appropriate distance, so that there is no overlapping when placed in the stereoscope, a picture in striking relief is obtained when combined together in the stereoscope. In this case it will be noticed that there is no portion common to both fields of view. In each case the overlapping portion is combined with white. It seems probable that this is how binocular vision takes place in ordinary circumstances. If an object in high relief-as, for instance, a vase or the face of a person-be viewed at a short distance and one particular point fixated, it will be noticed that the right eye dominates the right side of the field of vision and the left eye the left side. The image seen is almost entirely that of the right eye for the right side and that of the left eye for the left side, as may be proved by noticing the relation of surrounding objects, and closing first one eve and then the other alternately.

F. W. Edridge-Green.

\section{The Energy of Cyclones.}

IT does not seem to me as though any really satisfactory theory has yet been put forward to explain the genesis and maintenance of cyclones; I fully agree with $\mathrm{Mr}$. Deeley (November II, p. 345) that thev are not due to contiguous masses of air at different temperatures, but, on the other hand, I do not see how they can originate in an inert and stable region like the stratosphere.

Were storms produced by contrasts of temperature -or, in other words, by the so-called polar frontsurely they would be most violent where the contrast was most marked. The stormiest parts of the world are the great belt of the southern ocean from $40^{\circ}$ to $60^{\circ} \mathrm{S}$. lat. and that part of the Atlantic which lies north-west of Scotland, and neither of these regions shows any exceptionally steep gradient of temperature.

Observations in the upper air have shown a remarkable uniformity in the mean temperature (mean with regard to height) from 0 to $20 \mathrm{~km}$. in every place where they have been obtained, and it follows as a corollary that there is a very uniform pressure at $20 \mathrm{~km}$. height over the globe, for the pressure at $20 \mathrm{~km}$. is almost independent of the surface pressure. 